\title{
PARP9 is overexpressed in human breast cancer and promotes cancer cell migration
}

\author{
XINGHONG TANG ${ }^{1}$, HONGYING ZHANG $^{2}$, YAN LONG ${ }^{2}$, HUI HUA $^{3}$, YANGFU JIANG ${ }^{2}$ and JING JING ${ }^{1}$ \\ ${ }^{1}$ Department of Thyroid and Breast Surgery, ${ }^{2}$ Laboratory of Oncogene and ${ }^{3}$ Laboratory of Stem Cell Biology, \\ West China Hospital, Sichuan University, Chengdu, Sichuan 610041, P.R. China
}

Received January 20, 2017; Accepted June 1, 2018

DOI: $10.3892 / \mathrm{ol} .2018 .9124$

\begin{abstract}
Poly(ADP-Ribose) polymerase family member 9 (PARP9) promotes the proliferation, survival and chemotherapy resistance in lymphoma and prostate cancer. The expression and function of PARP9 in human breast cancer remains unknown. In the present study, it was demonstrated that PARP9 is frequently overexpressed in human breast cancer. In 57 normal breast tissues, the expression of PARP9 was not detected in 43 cases $(75.4 \%)$, but low levels of PARP9 were detected in 13 cases $(22.8 \%)$, and modest levels of PARP9 (PARP9/GAPDH ratio 1:1) were detected in only 1 case $(1.7 \%)$. In contrast, the expression of PARP9 was detected in all 57 breast cancer tissues, in which the levels of PARP9 were higher than that in paired normal breast tissues. In addition, high levels of PARP9 were detected in $43.8 \%$ of breast cancer tissues. Overexpression of PARP9 was negatively associated with estrogen receptor expression, and positively associated with axillary lymph node metastasis. However, PARP9 expression was not associated with other clinicopathological parameters, including age, HER-2 and tumor size. Furthermore, PARP9-knockdown inhibited breast cancer cell migration. These data indicate that PARP9 may promote breast cancer progression.
\end{abstract}

\section{Introduction}

Breast cancer is the most common type of cancer in women (1). Although the prognosis of human breast cancer can be significantly improved by surgical therapy, radiotherapy, chemotherapy, endocrine therapy and molecular targeted therapy, breast cancer incidence has increased in recent decades (2-5). Breast cancer comprises a group of heterogeneous diseases, whose prognoses differ due to variation in molecular

Correspondence to: Dr Jing Jing, Department of Thyroid and Breast Surgery, West China Hospital, Sichuan University, 37 Guo Xue Xiang, Chengdu, Sichuan 610041, P.R. China E-mail: jj_zcy@vip.163.com

Key words: Poly(ADP-Ribose) polymerase family member 9, breast cancer, metastasis and pathological characteristics (6). Treatment of breast cancer is based on various clinicopathological parameters, including the status of lymph node metastasis and hormone receptor expression $(7,8)$. Invasion of the lymph nodes implicates that tumor cells may spread, and combined therapies are required (9). Furthermore, the expression of estrogen receptor (ER) provides a basis for endocrine therapy (10).

Numerous oncogenes are involved in the occurrence and progression of breast cancer. PARP is a family of proteins involved in cellular processes, including DNA repair and apoptosis (11). The most well-established PARP protein is PARP1, which can be cleaved and inactivated by caspase during apoptosis (12). Notably, PARP9 lacks PARP activity, despite having similar carboxyl-terminal amino acid sequences to other members of the PARP family (13). Previous studies suggest that PARP9 may promote metastasis in a variety of tumors. PARP9 is overexpressed in lymphoma and prostate cancer and has been demonstrated to positively correlate with metastasis, recurrence and chemotherapy resistance $(14,15)$. However, the expression of PARP9 and its implication in breast cancer remain elusive. In the present study, the expression of PARP9 in 57 pairs of breast cancer and normal breast tissues was investigated, as well as the association between PARP9 and clinicopathological parameters. Furthermore, the effects of PARP9 on breast cancer-cell migration were detected.

\section{Materials and methods}

Tissues samples. A total of 57 pairs of breast cancer and normal breast tissues were obtained from the tissue bank at West China Hospital (Chengdu, China). All patients were female, who underwent surgery between February 2010 and May 2015. The diagnosis of breast cancer was confirmed by two patologists at West China Hospital, following biopsy. The expression of human epithelial growth factor receptor-2 (Her-2) was defined as positive by immunohistochemistry (3+) or fluoresence in situ hybridisation examination (+). Ki-67 levels were considered high if the percentage was $\geq 14 \%$ detected by immunohistochemistry.

No radiotherapy or chemotherapy had been received prior to operation. The age of patients ranged from 25-81 years, with a median age of 52 years. There were 54 cases of invasive ductal carcinoma, 2 cases of invasive lobular carcinoma and only 1 case of mucinous carcinoma. 


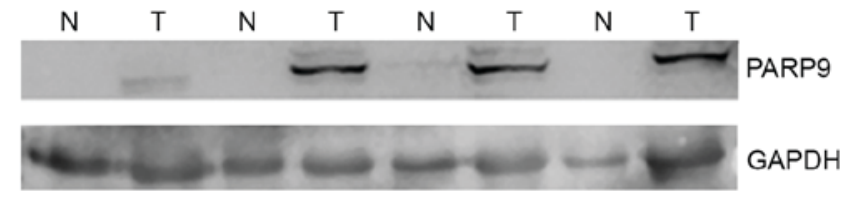

Figure 1. The expression of PARP9 in paired breast cancer and normal breast tissues. Cell lysates from breast cancer and normal breast samples were subjected to western blot analysis of PARP9 expression. PARP9, Poly(ADP-Ribose) polymerase family member 9; N, normal breast tissue; $\mathrm{T}$, breast cancer tissue.

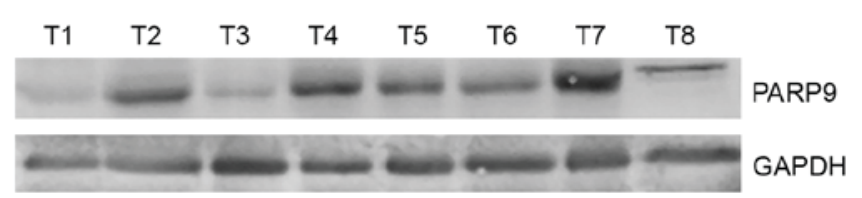

Figure 2. The expression of PARP9 in multiple breast cancer samples. Cell lysates from breast cancer samples were subjected to western blot analysis of PARP9 expression. PARP9, Poly(ADP-Ribose) polymerase family member 9; $\mathrm{T}$, breast cancer tissue.

Reagents. The polyclonal rabbit anti-human PARP9 antibody (cat. no. 17535-1-AP) was purchased from Proteintech, Inc. (Wuhan, China). The monoclonal mouse GAPDH (cat. no. 200306) and actin (cat. no. 250132) antibodies, and horseradish peroxidase-conjugated goat anti-mouse IgG (cat. no. 511103) and goat anti-rabbit IgG (cat. no. 511203) secondary antibodies were purchased from Zen Bioscience Co., Ltd. (Chengdu, China).

Western blot analysis. Tissues were lysed with ice-cold lysis buffer containing 1\% Triton X-100, 40 mM Hepes pH 7.5, $120 \mathrm{mM} \mathrm{NaCl}, 1 \mathrm{mM}$ EDTA, $10 \mathrm{mM}$ pyrophosphate, $10 \mathrm{mM}$ glycerophosphate, $50 \mathrm{mM} \mathrm{NaF}, 0.5 \mathrm{mM}$ orthovanadate, PMSF and aprotinin (Roche Diagnostics, Basel, Switzerland). Tissue lysates were ground for $30 \mathrm{sec} /$ time (4 times) until the tissues were totally triturated, then the mixture were incubated on ice for $30 \mathrm{~min}$ and centrifuged for $15 \mathrm{~min}$ at $14,000 \mathrm{x}$ g to remove cell debris. The supernatants were harvested and protein concentration was using a BCA assay (Thermo Fisher Scientific, Inc., Waltham, MA, USA). The proteins were heated at $100^{\circ} \mathrm{C}$ in 4X loading buffer [containing $200 \mathrm{nM}$ Tri-HCl, $40 \%$ glycerol, $10 \% \operatorname{SDS}(\mathrm{w} / \mathrm{v}), 400 \mathrm{nM}$ DTT, 0.04\% Bromphenil blue (w/v)] for $10 \mathrm{~min}$, samples containing $20 \mu \mathrm{g}$ protein were resolved by $10 \%$ SDS-PAGE, then transferred to a polyvinylidene difluoride membrane (Millipore; Merck KGaA, Darmstadt, Germany). Each membrane was blocked with 5\% non-fat dry milk in Tris buffered saline with Tween-20 (TBST) for $1 \mathrm{~h}$ at room temperature. The membranes were then incubated with PARP9 $(1: 1,500)$, actin $(1: 1,000)$ and GAPDH $(1: 1,000)$ primary antibodies overnight at $4^{\circ} \mathrm{C}$ and the appropriate secondary antibodies $(1: 1,500)$ for $1 \mathrm{~h}$ at room temperature. Detection was performed using a chemiluminescence kit (BeyoECL plus; cat. no. P0018; Beyotime Biotechnology, Shanghai, China). Images were collected by Alpha Innotech's FluorChem imaging system (Alpha Innotech, San Leandro, CA, USA). GAPDH was used as an internal control. Western blots were subjected to densitometric analysis using Quantity One software (version 4.6.2; Bio-RAD, Hercules, CA, USA). The PARP9/GAPDH ratio in
Table I. The association between PARP9 expression and clinicopathological parameters.

\begin{tabular}{|c|c|c|c|}
\hline $\begin{array}{l}\text { Clinicopathological } \\
\text { parameters }\end{array}$ & $\begin{array}{l}\text { PARP9 }{ }^{\text {high }} \\
\quad(n=25)\end{array}$ & $\begin{array}{l}\text { PARP9low } \\
(n=32)\end{array}$ & P-value \\
\hline Age (years) & & & 0.108 \\
\hline$\leq 45$ & 8 & 18 & \\
\hline$>45$ & 17 & 14 & \\
\hline ER status & & & 0.017 \\
\hline Positive & 11 & 24 & \\
\hline Negative & 14 & 8 & \\
\hline PR status & & & 0.117 \\
\hline Positive & 14 & 11 & \\
\hline Negative & 11 & 21 & \\
\hline Her-2 status & & & 0.592 \\
\hline Positive & 12 & 14 & \\
\hline Negative & 11 & 18 & \\
\hline $\begin{array}{l}\text { Axillary lymph } \\
\text { node metastasis }\end{array}$ & & & 0.007 \\
\hline Positive & 19 & 12 & \\
\hline Negative & 6 & 20 & \\
\hline $\mathrm{Ki}-67$ index $(\%)$ & & & 0.135 \\
\hline$\leq 14$ & 4 & 11 & \\
\hline$>14$ & 21 & 20 & \\
\hline Tumor size $(\mathrm{cm})$ & & & 0.513 \\
\hline$\leq 2$ & 4 & 6 & \\
\hline$>2$ & 21 & 25 & \\
\hline Stage & & & 0.575 \\
\hline $\mathrm{I} / \mathrm{II}$ & 16 & 23 & \\
\hline III/IV & 9 & 9 & \\
\hline Molecular type & & & 0.087 \\
\hline Luminal A & 2 & 8 & \\
\hline Luminal B & 9 & 13 & \\
\hline Her-2-enriched & 7 & 2 & \\
\hline Basal-like & 7 & 7 & \\
\hline
\end{tabular}

PARP9, Poly(ADP-Ribose) polymerase family member 9; ER, estrogen receptor; PR, progesterone receptor; Her-2, Human epidermal growth factor receptor 2 .

all breast cancer samples was calculated. The level of PARP9 expression was considered high if the PARP9/GAPDH ratio exceeded the median value in these samples.

Cell culture. The breast cancer cell line, HCC1806, was a gift from Professor Ceshi Chen (Kunming Institute of Zoology, Chinese Academy of Sciences, Shanghai, China). The cells were maintained in Dulbecco's minimal essential medium (Life Technologies, Grand Island, NY, USA) containing 10\% fetal bovine serum (Thermo Fisher Scientific, Inc.). Cells were incubated at $37^{\circ} \mathrm{C}$ in a humidified atmosphere with $5 \% \mathrm{CO}_{2}$.

RNA interference. All small interfering RNAs (siRNAs) were synthesized by GenePharma, Co, Ltd. (Shanghai, 
A

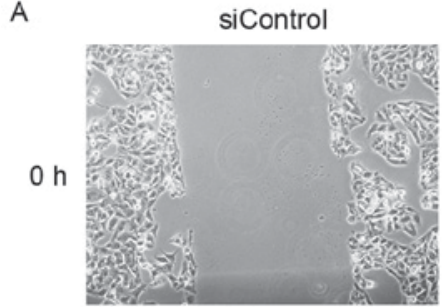

$48 \mathrm{~h}$

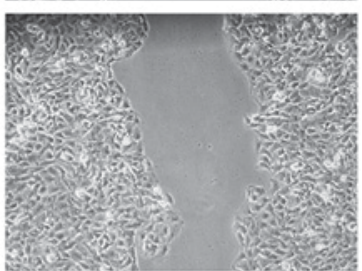

$72 \mathrm{~h}$

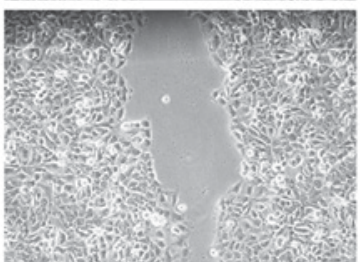

$96 \mathrm{~h}$

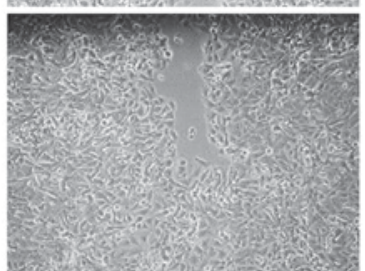

si-PARPg
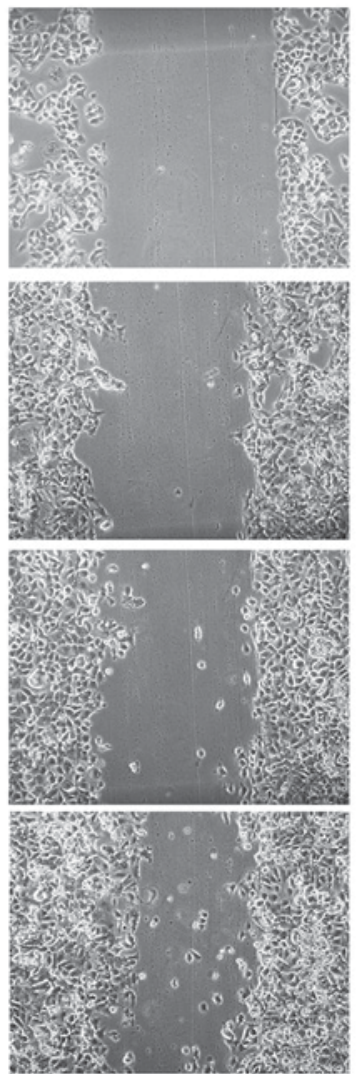

B

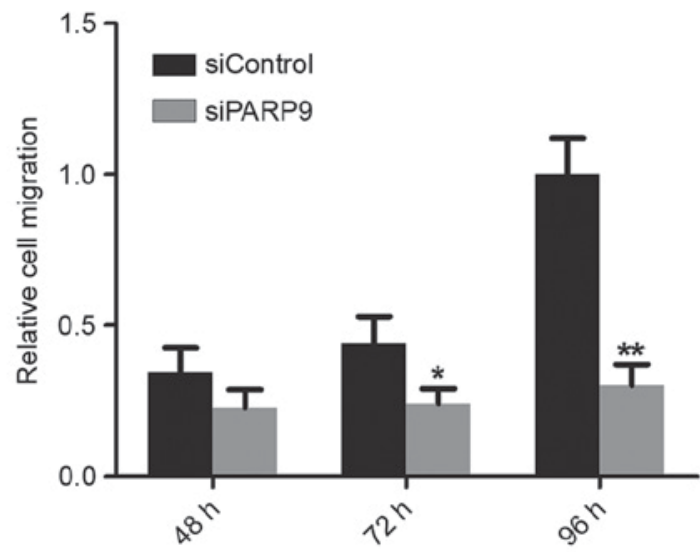

C

siControl siPARPg

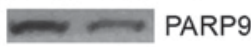

Figure 3. The effect of PARP9-knockdown on breast cancer cell migration. HCC1806 cells were transfected with control siRNA or PARP9 siRNA, followed by wound-healing assays. (A) The effects of PARP9 on cell migration. (B) The relative migration rate at different time points. The migration rate for siControl-transfected cells at $96 \mathrm{~h}$ was set as $1 .{ }^{*} \mathrm{P}<0.05 ;{ }^{* *} \mathrm{P}<0.001$. (C) The efficiency of PARP9-knockdown was confirmed by western blotting. PARP9, Poly(ADP-Ribose) polymerase family member 9; si, small interfering RNA.

China). The target sequence used for knockdown of PARP9 was 5'-GGACAGAGTTAGAGATTGAAAC-3', and the sequence of si-Control was 5'-ACGUGACACGUUCGGAGA ATT-3'. siRNA $(10 \mu \mathrm{M})$ were transfected into HCC1806 cells $(\sim 7,000$ cells per well) by Lipofectamine RNAimax reagent (Thermo Fisher Scientific, Inc.), according to the manufacturer's protocol. Cells were cultured for $48 \mathrm{~h}$ prior to subsequent western blotting.

Wound-healing assay. Cells were seeded in 6-well plates at 60,000 cells/well. The cells were cultured in DMEM medium containing $5 \%$ fetal bovine serum for $24 \mathrm{~h}$. To inhibit cell proliferation, the cells were treated with $2 \mu \mathrm{g} / \mathrm{ml}$ mitomycin $\mathrm{C}$ at $37^{\circ} \mathrm{C}$ for $96 \mathrm{~h} .24 \mathrm{~h}$ after the transfection of si-PARP9 or si-Control, a 1-mm scratch was made in the confluent cultures with a pipette tip, and washed twice with PBS to remove debris. The area of the scratch was measured by taking images under a phase-contrast microscope. To ensure the consistency of observation, specific observing points along the wound were labeled, and the same fields were observed at multiple time points.

Statistical analysis. Statistical analysis was conducted using SPSS software (version 17.0; SPSS, Inc., Chicago, IL, USA) and Graphpad Prism software (version 5.0; GraphPad Software, Inc., La Jolla, CA, USA) was used to produce graphs. Fisher's exact test was applied to determine statistical significance on the relationship between PARP9 and clinicopathological parameters. Student's t-test was used to determine the statistical difference in migration rate between siControl- and siPARP9-transfected cells. $\mathrm{P}<0.05$ was considered to indicate a statistically significant difference.

\section{Results}

PARP9 is frequently overexpressed in human breast cancer. PARP9 expression was not detected in 43 normal breast tissues (75.4\%), whereas it was detected in all 57 breast cancer tissues. Low levels of PARP9 (PARP9/GAPDH ratio $<10 \%$ of the median value in cancer tissues) were detected in 13 cases (22.8\%), and modest levels of PARP9 (PARP9/GAPDH ratio $\sim 1: 1)$ were detected in only 1 case $(1.7 \%)$. In all 57 cases, the levels of PARP9 in breast cancer tissues were higher than that in paired normal breast tissues (Fig. 1). In 57 cases of breast cancer tissues, high levels of PARP9 were detected in 25 cases $(43.8 \%)$, while low levels of PARP9 were detected in 32 cases (56.1\%) (Fig. 2).

PARP9 expression is associated with lymph node metastasis. In 57 breast cancer tissues, 35 cases were ER $\alpha$-positive, while 22 cases were ER $\alpha$-negative. Overexpression of PARP9 was negatively associated with $\mathrm{ER} \alpha$ expression (Table I). Axillary lymph nodes metastasis was detected in 31 cases (54.4\%), and high levels of PARP9 were detected in 25 cases 
(43.8\%) of breast cancer. Overexpression of PARP9 was positively associated with axillary lymph nodes metastasis $(\mathrm{P}<0.01$; Table I). PARP9 expression was not associated with age, Her-2 expression, Ki-67 or tumor size ( $\mathrm{P}>0.05$; Table I).

PARP9-knockdown inhibits breast cancer-cell migration. Since overexpression of PARP9 was positively associated with lymph node metastasis, the effects of PARP9 on breast cancer-cell migration were investigated. PARP9 siRNA was transfected into HCC1806 cells, followed by a wound-healing assay. The efficiency of PARP-knockdown was confirmed by western blotting, and it was demonstrated that PARP9-knockdown significantly inhibited HCC1806 cell migration (Fig. 3).

\section{Discussion}

The progression of breast cancer involves aberrant expression of hormone receptors, growth factor receptors and other oncogenes, including mechanistic target of rapamycin kinase $(16,17)$. In the present study, it was demonstrated that PARP9 was overexpressed in $43.8 \%$ of human breast cancer cases. Overexpression of PARP9 was negatively associated with ER expression, but positively associated with axillary lymph node metastasis. PARP9-knockdown was indicated to inhibit breast cancer cell migration. These data suggest that PARP9 may promote breast cancer progression.

A previous study demonstrated that PARP9 is involved in the interferon (IFN)- $\gamma$-signal transducer and activator of transcription 1 (STAT1) pathway. PARP9 interacts with STAT $1 \alpha$ and STAT1 $\beta$, upregulates the expression of interferon regulatory factor (IRF) 2 and downregulates that of IRF1, thereby promoting cell proliferation, survival and migration (18). Also, PARP9 has been demonstrated to promote recurrence, metastasis and chemotherapy-resistance in prostate cancer and lymphoma $(14,15)$. The expression of PARP9 has been demonstrated to be more abundant in the androgen-resistant prostate cell lines, PC3 and DU145, compared with the AR-dependent cell line, LNCap, and normal prostate epithelial cell line, SUDHL7 (15). PARP9 has been indicated to enhance tumor cell survival and doxorubicin-resistance via the E3 ubiquitin ligase, DTX3L, which selectively ubiquitinates histone $\mathrm{H} 4$ and protects cells against DNA-damaging agents following binding to PARP in chemotherapy-resistant diffuse large B cell lymphoma (19).

Although STAT1 has been established to have anti-tumor effects, increasing the expression of STAT1 and IFN- $\gamma$-induced genes contributed to metastatic potential by enhancing the ability of malignant cells to invade the stroma and migrate to distant sites in node-positive triple negative breast tumors (20). PARP9 has also been demonstrated to form complex with STAT1 and DTX3L, which may affect the nuclear activities of STAT1 by antagonistically regulating the tyrosine phosphorylation of STAT1 on Y701 (21). In consistence with previous studies on PARP9 in prostate cancer, the present study demonstrates that PARP9 promotes breast cancer cell migration. Thus, overexpression of PARP9 may promote the progression of human breast cancer.

\section{Acknowledgements}

Not applicable.

\section{Funding}

No finding was received.

\section{Availability of data and materials}

The datasets used and/or analyzed during the current study are available from the corresponding author on reasonable request.

\section{Authors' contributions}

$\mathrm{XT}, \mathrm{JJ}$ and $\mathrm{YJ}$ conceived the study and wrote the manuscript. $\mathrm{XT}$ and $\mathrm{HZ}$ performed the experiments. YL and $\mathrm{HH}$ assisted in collecting and analyzing the patient data regarding the clinicopathological parameters of breast cancer. All authors read and approved the final manuscript.

\section{Ethics approval and consent to participate}

The study was approved by the ethics committee of West China Medical Center, Sichuan University and informed consent to participate was signed by the patients and/or guardians.

\section{Patient consent for publication}

Informed consent was signed by the patients and/or guardians.

\section{Competing interests}

The authors declare that they have no competing interests.

\section{References}

1. Xiang M, Su H, Shu G, Wan D, He F, Loaec M, Ding Y, Li J, Dovat S, Yang G and Song C: Amplexicaule A exerts anti-tumor effects by inducing apoptosis in human breast cancer. Oncotarget 7: 18521-18530, 2016

2. Early Breast Cancer Trialists' Collaborative Group (EBCTCG), Peto R, Davies C, Godwin J, Gray R, Pan HC, Clarke M, Cutter D, Darby S, McGale P, et al: Comparisons between different polychemotherapy regimens for early breast cancer: Meta-analyses of long-term outcome among 100,000 women in 123 randomised trials. Lancet 379: 432-444, 2012.

3. Zeng H, Zheng R, Zhang S, Zou X and Chen W: Female breast cancer statistics of 2010 in China: Estimates based on data from 145 population-based cancer registries. J Thorac Dis 6: 466-470, 2014.

4. Azim HA Jr, de Azambuja E, Colozza M, Bines J and Piccart MJ: Long-term toxic effects of adjuvant chemotherapy in breast cancer. Ann Oncol 22: 1939-1947, 2011.

5. EBCTCG (Early Breast Cancer Trialists' Collaborative Group), McGale P, Taylor C, Correa C, Cutter D, Duane F, Ewertz M, Gray R, Mannu G, Peto R, et al: Effect of radiotherapy after mastectomy and axillary surgery on 10-year recurrence and 20-year breast cancer mortality: Meta-analysis of individual patient data for 8135 women in 22 randomised trials. Lancet 383 : 2127-2135, 2014.

6. Wang XL, Tao L, Zhou XL, Wei H and Sun JW: Initial experience of automated breast volume scanning (ABVS) and ultrasound elastography in predicting breast cancer subtypes and staging. Breast 30: 130-135, 2016. 
7. Selli C, Dixon JM and Sims AH: Accurate prediction of response to endocrine therapy in breast cancer patients: Current and future biomarkers. Breast Cancer Res 18: 118, 2016.

8. Andree C, Schmidt VJ, Munder BIJ, Seidenstïcker K, Behrendt P, Witzel C, Horch RE, Andrews BT and Richrath P: Detecting of breast cancer metastasis by means of regional lymph node sampling during autologous breast reconstruction-a screening of 519 consecutive patients. Med Sci Monit 18: CR605-CR610, 2012.

9. Nicolini A, Ferrari P and Duffy MJ: Prognostic and predictive biomarkers in breast cnacer: Past, present and future. Semin Cancer Biol pii: 2017 (Epub ahead of print).

10. Thomas $C$ and Gustafsson JA: The different roles of ER subtypes in cancer biology and therapy. Nat Rev Cancer 11: 597-608, 2011

11. Lehmann M, Pirinen E, Mirsaidi A, Kunze FA, Richards PJ, Auwerx J and Hottiger MO: ARTD1-induced poly-ADP-ribose formation enhances PPAR $\gamma$ ligand binding and co-factor exchange. Nucleic Acids Res 43: 129-142, 2015.

12. Qin WD, Liu GL, Wang J, Wang H, Zhang JN, Zhang F, Ma Y, Ji XY, Li C and Zhang MX: Poly(ADP-ribose) polymerase 1 inhibition protects cardiomyocytes from inflammation and apoptosis in diabetic cardiomyopathy. Oncotarget 7: 35618-35631, 2016.

13. Iwata H, Goettsch C, Sharma A, Ricchiuto P, Goh WW, Halu A, Yamada I, Yoshida H, Hara T, Wei M, et al: PARP9 and PARP14 cross-regulate macrophage activation via STAT1 ADP-ribosylation. Nat Commun 7: 12849, 2016.

14. Juszczynski P, Kutok JL, Li C, Mitra J, Aguiar RC and Shipp MA: BAL1 and BBAP are regulated by a gamma interferon-responsive bidirectional promoter and are overexpressed in diffuse large B-cell lymphomas with a prominent inflammatory infiltrate. Mol Cell Biol 26: 5348-5359, 2006.
15. Bachmann SB, Frommel SC, Camicia R, Winkler HC, Santoro R and Hassa PO: DTX3L and ARTD9 inhibit IRF1 expression and mediate in cooperation with ARTD8 survival and proliferation of metastatic prostate cancer cells. Mol Cancer 13: 125, 2014.

16. Zhou X, Tan M, Hawthorne VS, Klos KS, Lan KH, Yang Y, Yang W, Smith TL, Shi D and Yu D: Activation of the Akt/mammalian target of rapamycin/4E-BP1 pathway by ErbB2 overexpression predicts tumor progression in breast cancers. Cancer Res 10: 6779-6788, 2004.

17. Yin Y, Hua H, Li M, Liu S, Kong Q, Shao T, Wang Y, Luo Q, Wang J, Luo T and Jiang Y: mTORC2 promotes type I insulin-like growth factor receptor and insulin receptor activation through the tyrosine kinase activity of mTOR. Cell Res 26: 46-65, 2016.

18. Camicia R, Bachmann SB, Winkler HC, Beer M, Tinguely M, Haralambieva E and Hassa PO: BAL1/ARTD9 represses the antiproliferative and pro-apoptotic IFNgamma-STAT1-IRF1-p53 axis in diffuse large B-cell lymphoma. J Cell Sci 126: 1969-1980, 2013.

19. Zhang Y,Mao D, Roswit WT, Jin X, Patel AC, Patel DA, Agapov E, Wang Z, Tidwell RM, Atkinson JJ, et al: PARP9-DTX3L ubiquitin ligase targets host histone $\mathrm{H} 2 \mathrm{BJ}$ and viral $3 \mathrm{C}$ protease to enhance interferon signaling and control viral infection. Nat Immunol 16: 1215-1227, 2015.

20. Greenwood C, Metodieva G, Al-Janabi K, Lausen B, Alldridge L, Leng L, Bucala R, Fernandez N and Metodiev MV: Statl and CD74 overexpression is co-dependent and linked to increased invasion and lymph node metastasis in triple-negative breast cancer. J Proteomics 75: 3031-3040, 2012.

21. Yan Q, Xu R, Zhu L, Cheng X, Wang Z, Manis J and Shipp MA: BAL1 and its partner E3 ligase, BBAP, link poly(ADP-ribose) activation, ubiquitylation, and double-strand DNA repair independent of ATM, MDC1, and RNF8. Mol Cell Biol 33: 845-857, 2013. 